\title{
An integrative module analysis of DNA methylation landscape in aging
}

\author{
GANG LI, KE-YU LIU and ZHONG-PENG QIU \\ Department of Orthopedics, School of Medicine, Shihezi University, Shihezi, Xinjiang 832000, P.R. China
}

Received June 26, 2018; Accepted February 6, 2019

DOI: $10.3892 / \mathrm{etm} .2019 .7334$

\begin{abstract}
To investigate the molecular mechanism of aging, the combination of module analysis and DNA methylation data was used to detect dynamically controlled modules for aging. Multiple differential expression networks (DENs) were constructed based on the microarray profiles across different aging groups ( $<70$ years, $70-80$ years, and $>80$ years) Next, a module-based approach was utilized to extract the common candidate modules across all age groups. We used Module Connectivity Dynamic Score (MCDS) to quantify the connectivity change of the common modules among the different age groups. Functional analyses were implemented for the genes in the common modules to further identify the significant biological processes. A total of two DENs were constructed. Overall 657 informative genes were screened out. When false discovery rate (FDR) was set as 0.05 , we found that 148 modules were significant. Only 1 significant 2-differential modules (DMs) (module 493) with dynamic changes was discovered. Significantly, the genes in the module 493 participated in 7 significant pathways, including pentose phosphate pathway, carbon metabolism, and citrate cycle (TCA cycle). In conclusion, pathway functions [pentose phosphate pathway, carbon metabolism, citrate cycle (TCA cycle), chromosomal instability, ateroid biosynthesis, PPAR signaling pathway, and immune response] may serve as potential therapeutic targets in aging.
\end{abstract}

\section{Introduction}

Aging is a natural as well as undeniable developmental process in human life, and 'biological age' is usually affected by many factors including environment, heredity, lifestyle, as well as any kind of disease (1). Developing reliable age-related measures is the main goal of gerontology (2). Biomarkers related to

Correspondence to: Dr Gang Li, Department of Orthopedics, School of Medicine, Shihezi University, 107 Bei'er Road, Shihezi, Xinjiang 832000, P.R. China

E-mail: lig1993@163.com

Key words: aging, informative genes, module, pathways, DNA methylation biological age have been identified in the research to detect the age of a person, and these signatures are significant for geriatric evaluation, which might be in favor of the adaptation of habits to assist healthy aging $(3,4)$. Many aging biomarkers have been investigated, for example, telomere length, and mitochondrial DNA $(5,6)$. Nevertheless, all of these signatures have a relatively low precision (7).

Methylated DNA is biologically and chemically more stable in relevance to mRNA. DNA methylation (DNAm) has been demonstrated to be an especially promising signature of aging (8-10). Bekaert et al (11) have predicted the person's age based on four age-associated DNAm biomarkers. Previous studies have demonstrated that the age-related epigenetic drift may be closely related to disease progression (12) and human evolution (13). Therefore, it is of great interest and importance to uncover the specific dynamics of DNAm landscape in aging.

As known, complicated diseases are believed to be induced by the perturbations of biological networks, other than single genes. Nevertheless, in a previous research, only two conditions were considered (that is to say, there is only one biological network) (14). Therefore, simultaneously measuring network dynamics in the progression of a disease is very important to understand the molecular mechanisms underlying the given disease. Of note, with the development of high throughput techniques, a great deal of protein interactions are collected, however, a number of interactions are yet not measured (15). This problem might be solved, to some extent, by utilizing modules or sub-networks within the complicated network (16). Hence, it is crucially important to detect significant modules in order to better understand the biological events related to aging.

In the present study, with the goal of detecting dynamically controlled modules related to aging, inference of multiple differential modules (iMDM) was utilized to analyze the DNA methylation data of aging at three age groups, in order to obtain the connectivity alterations of modules in the aging process. By utilizing iMDM to multiple sub-networks, candidate methylated genes were detected. We believe that our results can provide foundation for experimental verification in a future study, and expound the molecular mechanisms of aging.

\section{Materials and methods}

This module-based approach takes as input methylation data collected from control and disease cases, and is implemented 
based on the following steps: establishment of multiple differential expression networks (DENs) for each case; extraction of statistically significant multiple differential modules (DMs) in multiple DENs; quantification of the connectivity changes of the common multiple DMs; pathway and GO analyses for the genes in the common modules.

Microarray data. The microarray data of E-GEOD-64490 on aging were downloaded from the EMBL-EBI database (https://www.ebi.ac.uk/arrayexpress/experiments/E-GEOD64490/), relying on the A-GEOD-13534 - Illumina HumanMethylation450 BeadChip (HumanMethylation450_150174 82_v.1.1). In this study, gene microarray data of 48 samples were included. In the DNAm age, there were 14 samples with age $<70$ years, 29 samples of 70-80 years of age, and 5 samples of $>80$ years of age. After the probes were retrieved, we mapped the probes to the human gene symbols, and finally obtained 20,417 genes.

Protein-protein interaction network (PPIN) downloaded from STRING database. The human background PPIN covering 787,896 interactions and 16,730 genes was retrieved from STRING database (http://string-db.org/), and then only the common part of the microarray data and genes in the background PPIN were taken to construct the informative PPIN. Finally, 698,580 interactions were obtained.

Construction of DENs. For each age group, DEN was established based on the differential expression in the aging conditions. Firstly, on the basis of the absolute value of the Pearson's correlation coefficient (PCC) of any two genes, significant edges were selected to establish a binary co-expression network. Herein, we only selected the edges having PCC higher than the predefined value of 0.8 in order to construct the binary co-expression network. Secondly, we utilized one-side t-test to calculate the gene expressions in each age group. Using the P-value of differential gene expression in each age condition, we assigned the weight value to the interaction of the binary co-expression network. In view of multiple DENs, the same nodes were included but there were different edges; this was determined as $\mathrm{H}_{\mathrm{k}}=\left(\mathrm{V}, \mathrm{E}_{\mathrm{k}}\right)(1 \leq \mathrm{k} \leq \mathrm{M})$, where $\mathrm{V}$ is the node set of the co-expression network, $\mathrm{E}_{\mathrm{k}}$ is represented by a 3-dimensional matrix $A=\left(a_{i j k}\right) n \times n \times M$, where $a_{i j k}$ stands for the weight on the edge $\mathrm{e}(\mathrm{i}, \mathrm{j})$, in network $\mathrm{H}_{\mathrm{k}}$, and $\mathrm{M}$ denotes the number of DEN.

Identifying candidate modules across multiple DENs. The unique and common modules across multiple DENs (that is to say, multiple candidate modules) were identified. Module algorithm (17) was developed to select gene modules with the same gene content, but different connectivity among multiple interaction networks. This module algorithm was applied to extract the candidate modules. The specific steps are described below.

Prioritization of informative genes. In order to obtain the informative genes, the genes in the multiple DENs were ranked using degree characteristics. Then, we obtained the sort order for each gene among all DENs based on the mean value of z-scores across all DENs. We selected and determined the top $10 \%$ genes as informative genes.
Module identification. Starting with each informative gene, module identification iteratively included genes whose addition led to the maximum reduction in the graph entropy-based function until no reduction was found.

Refining candidate modules. We removed the multiple candidate modules having node sizes $<5$. Jaccard index was used to merge the overlapping multiple candidate modules. In the present analysis, we set the threshold of the Jaccard index $\geq 0.5$.

Statistical significance for multiple candidate modules. After obtaining the candidate modules, we implemented statistical significance for multiple candidate modules according to the null score distribution of candidate modules produced based on randomized networks. After that, Benjamini-Hochberg was utilized to adjust the P-values for multiple testing relying on false discovery rate (FDR) (18). FDR $<0.05$ was selected to be the significance threshold.

Connectivity dynamics of common multiple DMs and MCDS were used to further quantify the connectivity change of component modules. Analogously, we calculated the statistical significance of Module Connectivity Dynamic Score (MCDS) for a DM as that for multiple DMs. Concretely, we firstly computed the null distribution for MCDS scores according to the random multiple DMs. Then, we calculated the P-value for an MCDS on the basis of the null distribution. Benjamini-Hochberg was then used for correction. The DMs with greater connectivity were identified based on FDR $<0.05$.

Enrichment analysis for the genes in dynamic modules. To investigate the significant biological processes, DAVID was employed for pathway and GO annotation of genes in the dynamic modules. Expression Analysis Systematic Explorer (EASE) test was used to evaluate the significant pathways. Significant GO and pathway categories were determined according to FDR $<0.05$.

\section{Results}

Construction of multiple DENs. In addition to the age group $<70$ years, two conditions were also included, age group $70-80$ years, and age group $>80$ years, multiple $=2$. Thus, a total of two DENs were constructed, and 2-DMs were identified. Significant genes and biological processes were then extracted after the significance of DMs and MCDS was evaluated. These significant genes and biological processes across the two conditions might provide novel insights on the molecular mechanism of aging progression.

After we mapped the microarray data to the background PPIN, an informative PPIN was extracted, which included 15,248 genes. To increase the network confidence, only interactions with scores $\geq 0.8$ of the informative PPIN were selected in order to build DENs. Finally, we constructed two DENs, and each of them covered 87,702 edges, as well as 6,576 nodes. The DEN compositions are shown in Fig. 1.

Identification of multiple candidate modules in two DENs and statistical significance of candidate modules. Using the 
A

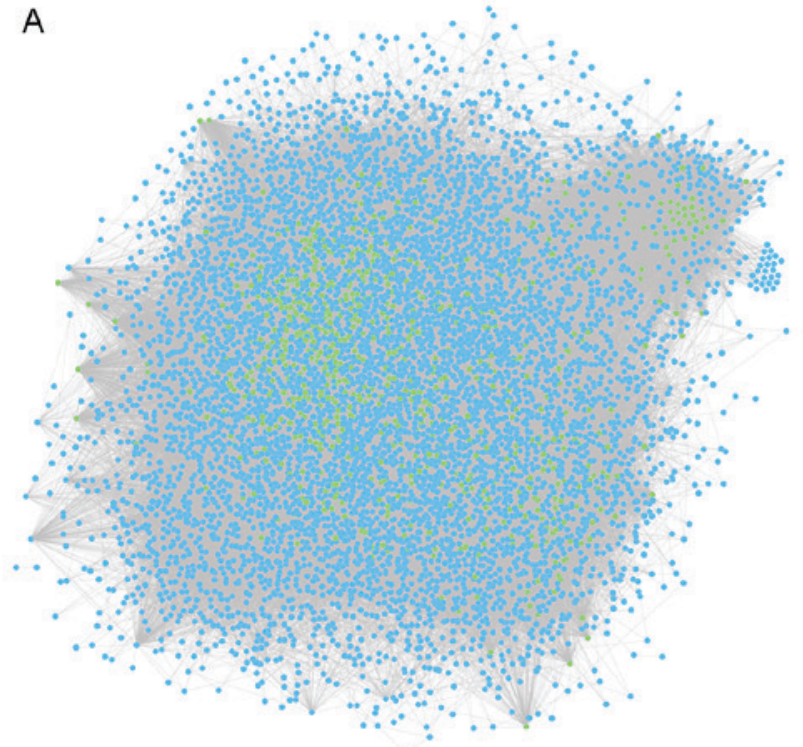

B

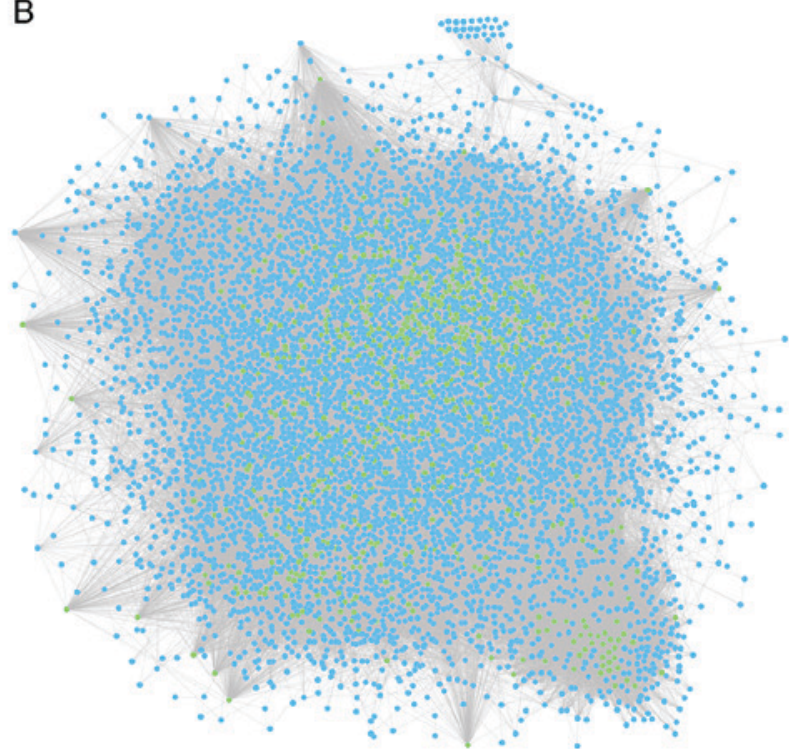

Figure 1. Compositions of DENs in comparison of the age groups (A) $<70$ years vs. $70-80$ years, and (B) $<70$ years vs. $>80$ years. Green nodes represent the informative genes. DEN, differential expression network.

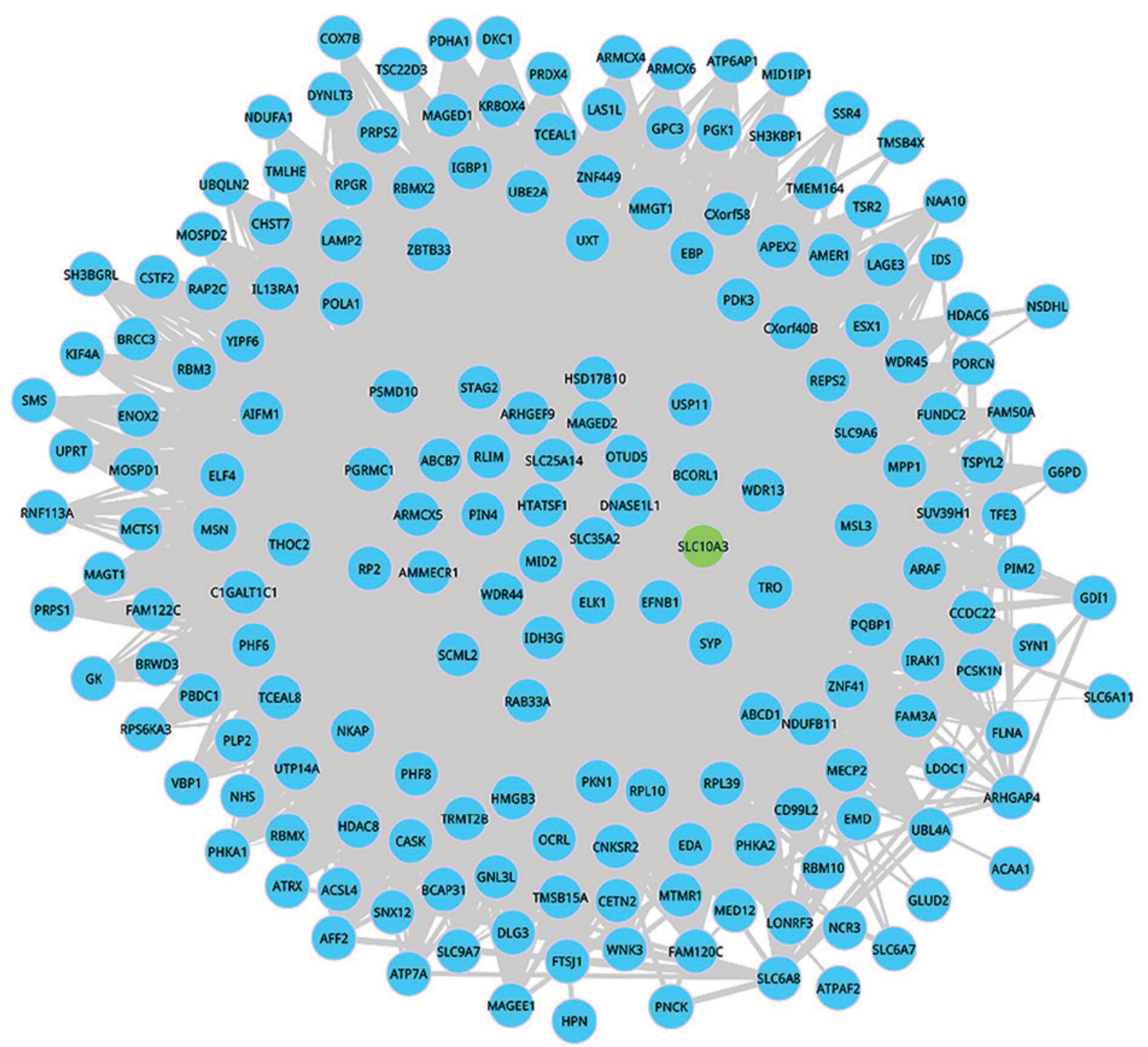

Figure 2. One dynamics 2-DMs identified in the age groups $<70$ years and $70-80$ years. Difference was calculated as ' $70-80$ years age group - $<70$ years age group. Green nodes represent the informative genes. 


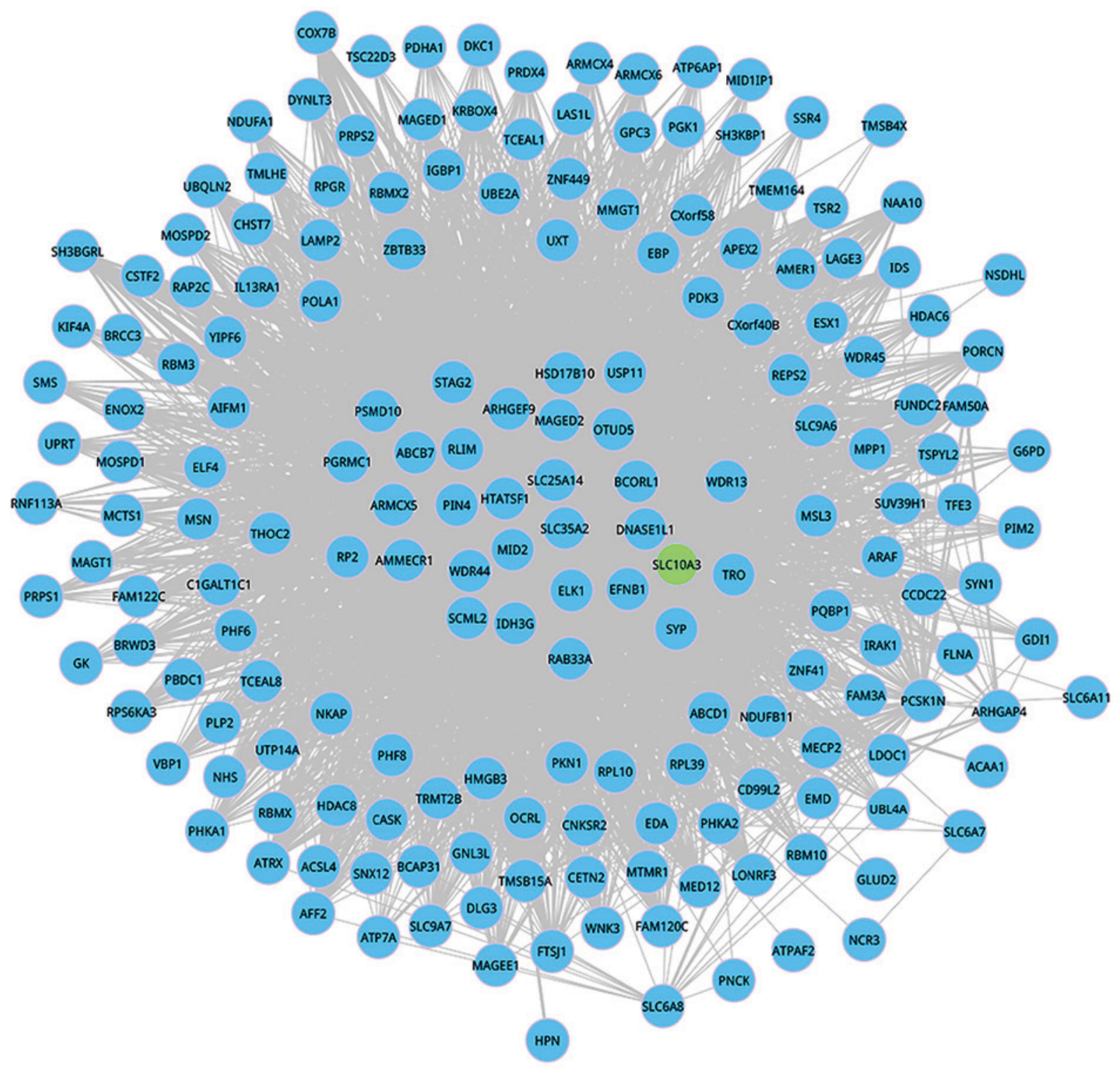

Figure 3. One dynamics 2-DMs identified in the age groups $<70$ years and $>80$ years. Difference was calculated as $>80$ years group - $<70$ years group'. Green nodes represent the informative genes.

z-score distribution of 6,576 nodes in the DENs, 657 informative genes were screened out. Afterwards, module search and refinement were conducted. Subsequently, a total of 162 candidate modules in the DENs of two aging conditions were identified. When FDR was set as 0.05 , we found that there were 148 significant modules.

Common 2-DMs used to uncover dynamics during the development of aging. When FDR was set as 0.05 , we found only module 493 observed in the DENs of aging group 70-80 years, and aging group $>80$ years was dynamic. This dynamic DM 493 including 187 nodes and 4,006 interactions is shown in Figs. 2 and 3. Among these 187 nodes, only one informative gene SLC10A3 appeared in this network. In the aging development, the connectivity of the interactions in this dynamic module was significantly changed, which further suggested that network rewired exerts key functions during the aging progression.
Because the weight of edges in the DENs is a measurement of evaluating the differential expression between control and disease, the average edge weight acts as a way to assess the differential activity of the module. Figs. 4 and 5 show the weight distribution of the interactions in the dynamic 2-DMs for the aging groups 70-80 years and $>80$ years, respectively. As shown in Fig. 4, the majority of interactions in the network of the aging group 70-80 years are distributed in the weight distribution of 0.3-0.4, 0.4-0.5, and 0.5-0.6. However, the majority of interactions in the network of the aging group $>80$ years are distributed in the weight distribution of $0-0.1$, and 0.1-0.2, as shown in Fig. 5.

Enrichment analysis. To investigate the biological processes, we implemented GO and pathway enrichment analysis for the genes in the dynamic module 493. Based on FDR $<0.05$, no GO terms were identified. However, overall 7 significant pathway terms were enriched in this dynamic module, including 


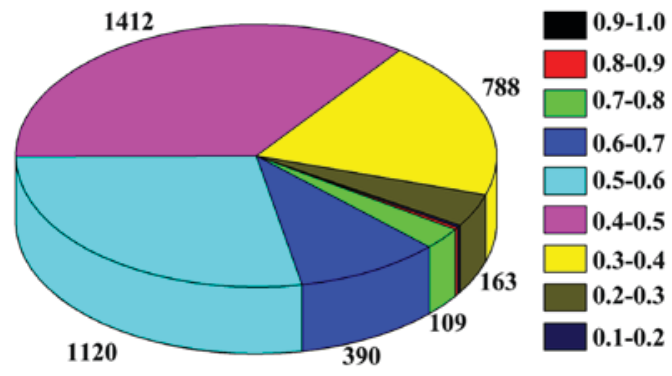

Figure 4. Weight distribution of the edges in the dynamic 2-DMs for the 70-80 years age group.

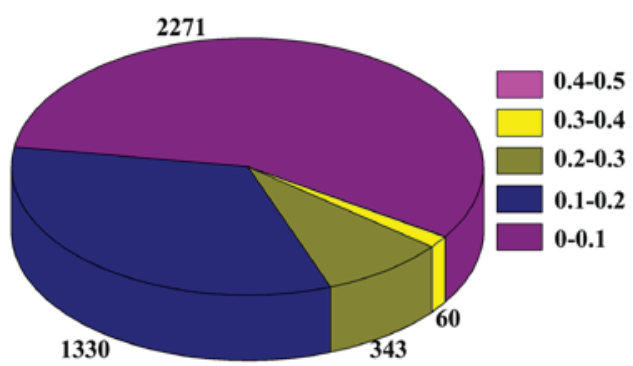

Figure 5. Weight distribution of the edges in the dynamic 2-DMs for the $>80$ years age group

pentose phosphate pathway, carbon metabolism, citrate cycle (TCA cycle), chromosomal instability, ateroid biosynthesis, PPAR signaling pathway, and immune response.

\section{Discussion}

Aging is a major risk factor for chronic, metabolic and neurodegenerative disorders. Developing reliable age-related modules and functional categories is the main goal of gerontology, helpful to understand the potential mechanisms and provide signatures for assisting healthy aging. In our study, in order to explore the molecular events indicative of aging, the available DNA methylation data and DEN were integrated to investigate the altered modules and biological functions related to aging. Significantly, 1 dynamic dysregulated module (module 493) was extracted GO enrichment results demonstrated that genes in module 493 were not involved in any category. Significantly, overall 7 significant pathway terms were enriched in the dynamic dysregulated module, including pentose phosphate pathway, carbon metabolism, citrate cycle (TCA cycle), chromosomal instability, ateroid biosynthesis, PPAR signaling pathway, and immune response.

Carbon metabolism that transforms carbon through pentose phosphate pathway, as well as TCA cycle to energy is essential for the physiology of brain, heart, liver, and kidneys (19). Significantly, mitochondria are responsible for ATP production, and progressive mitochondrial dysfunction, occurring with aging, leads to growing generation of reactive oxygen species (ROS), which in turn lead to further mitochondrial deterioration, as well as cellular damage (20). Furthermore, deletions and mutations of aged mitochondrial DNA have been reported to be related to aging (21). Of note, endurance training, as well as alternate-day-fasting has been suggested to enhance healthspan via avoiding mitochondrial degeneration (22). Thus, carbon metabolism, pentose phosphate pathway, and citrate cycle (TCA cycle) might exert significant functions in the progression of aging.

Chromosomal instability is one form of genomic instability, resulting from defects in chromosomal segregation, DNA damage, as well as telomere stability. Genetic evidence has demonstrated that the enhancement of the faithful chromosomal segregation can extend longevity in mammals (23). Telomere shortening is found in normal aging in mice and human (24). The premature aging of telomerase deficient mice can be reverted if telomerase is genetically reactivated in the aged mice (25). Accordingly, chromosomal instability is highly associated with aging progression.

Aging is implicated to be related to oxidative stress, which is regarded as the effector of the cascade of degenerative events (26). Oxidative stress is connected to the enhanced intracellular levels of ROS, and excessive production of ROS induces neuroinflammation, and neuronal death (27). Moderate increase of ROS has been demonstrated to serve as a molecular signal downstream effect which lure endogenous defense mechanisms, ending up with the increased stress resistance, as well as extended life span (28). Significantly, specific targets for a balanced ROS regulation are PPARs (29). In our study, the genes in the dynamic module participated in PPAR signaling pathway. Accordingly, PPAR signaling pathway might be highly related to the aging progression.

Immune system is an important component of the inflammatory process and is the natural response to infection. The recognition and elimination of senescent and hyperploid cells is an important role of the immune system (30). Inflammation plays important roles in the progression of obesity and diabetes, which are correlated with human aging (31). Overexpression of $\mathrm{NF}-\kappa \mathrm{B}$ pathway is observed in aging (32). Moreover, inhibiting $\mathrm{NF}-\kappa \mathrm{B}$ signaling pathway prevents age-related characteristics in mouse models of accelerated aging (33). Thus, we infer that aging might be modulated by $\mathrm{NF}-\kappa \mathrm{B}$-driven inflammatory responses.

Taken together, this study has established interesting management options (dynamic DMs and the corresponding pathways) for the assessing the quality of life in the aging population. However, this study had several limitations. The number of samples was small which might cause a high false-positive rate of the findings. Moreover, no experimental confirmation was done in vivo or in vitro based on patient tissue or an animal model. Thus, suture studies with larger number of samples, using an animal model or patient tissues, are needed to confirm our preliminary findings obtained from bioinformatics analysis.

\section{Acknowledgements}

Not applicable.

\section{Funding}

No funding was received.

\section{Availability of data and materials}

The datasets used and/or analyzed during the current study are available from the corresponding author upon reasonable request. 


\section{Authors' contributions}

GL was involved in the conception of the study, acquired the data and drafted the manuscript; KYL analyzed the data; ZPQ was involved in the conception of the study and revised the manuscript. All authors read and approved the final manuscript.

\section{Ethics approval and consent to participate}

Not applicable.

\section{Patient consent for publication}

Not applicable.

\section{Competing interests}

The authors declare that they have no competing interests.

\section{References}

1. Xu C, Qu H, Wang G, Xie B, Shi Y, Yang Y, Zhao Z, Hu L, Fang X, Yan J, et al: A novel strategy for forensic age prediction by DNA methylation and support vector regression model. Sci Rep 5: 17788, 2015.

2. Sanders JL, Boudreau RM, Penninx BW, Simonsick EM, Kritchevsky SB, Satterfield S, Harris TB, Bauer DC and Newman AB; Health ABC Study: Association of a Modified Physiologic Index with mortality and incident disability: The Health, Aging, and Body Composition Study. J Gerontol A Biol Sci Med Sci 67: 1439-1446, 2012.

3. Song Z, von Figura G, Liu Y, Kraus JM, Torrice C, Dillon P, Rudolph-Watabe M, Ju Z, Kestler HA, Sanoff H, et al: Lifestyle impacts on the aging-associated expression of biomarkers of DNA damage and telomere dysfunction in human blood. Aging Cell 9: 607-615, 2010.

4. Zbieć-Piekarska R, Spólnicka M, Kupiec T, Parys-Proszek A, Makowska Ż, Pałeczka A, Kucharczyk K, Płoski R and Branicki W: Development of a forensically useful age prediction method based on DNA methylation analysis. Forensic Sci Int Genet 17: 173-179, 2015.

5. Lin J, Epel E, Cheon J, Kroenke C, Sinclair E, Bigos M, Wolkowitz O, Mellon S and Blackburn E: Analyses and comparisons of telomerase activity and telomere length in human $\mathrm{T}$ and B cells: Insights for epidemiology of telomere maintenance. J Immunol Methods 352: 71-80, 2010.

6. Cortopassi GA, Shibata D, Soong NW and Arnheim N: A pattern of accumulation of a somatic deletion of mitochondrial DNA in aging human tissues. Proc Natl Acad Sci USA 89: 7370-7374, 1992 .

7. Meissner C and Ritz-Timme S: Molecular pathology and age estimation. Forensic Sci Int 203: 34-43, 2010.

8. Horvath S, Zhang Y, Langfelder P, Kahn RS, Boks MP, van Eijk K, van den Berg LH and Ophoff RA: Aging effects on DNA methylation modules in human brain and blood tissue. Genome Biol 13: R97, 2012.

9. Day K, Waite LL, Thalacker-Mercer A, West A, Bamman MM, Brooks JD, Myers RM and Absher D: Differential DNA methylation with age displays both common and dynamic features across human tissues that are influenced by $\mathrm{CpG}$ landscape. Genome Biol 14: R102, 2013.

10. Numata S, Ye T, Hyde TM, Guitart-Navarro X, Tao R, Wininger M, Colantuoni C, Weinberger DR, Kleinman JE and Lipska BK: DNA methylation signatures in development and aging of the human prefrontal cortex. Am J Hum Genet 90: 260-272, 2012.

11. Bekaert B, Kamalandua A, Zapico SC, Van de Voorde W and Decorte R: Improved age determination of blood and teeth samples using a selected set of DNA methylation markers. Epigenetics 10: 922-930, 2015.

12. Teschendorff AE, Jones A, Fiegl H, Sargent A, Zhuang JJ, Kitchener HC and Widschwendter M: Epigenetic variability in cells of normal cytology is associated with the risk of future morphological transformation. Genome Med 4: 24, 2012.
13. West J, Widschwendter M and Teschendorff AE: Distinctive topology of age-associated epigenetic drift in the human interactome. Proc Natl Acad Sci USA 110: 14138-14143, 2013.

14. MaX, Gao L, Karamanlidis G, Gao P,Lee CF, Garcia-Menendez L, Tian R and Tan K: Revealing pathway dynamics in heart diseases by analyzing multiple differential networks. PLoS Comput Biol 11: e1004332, 2015.

15. Nibbe RK, Chowdhury SA, Koyutürk M, Ewing R and Chance MR: Protein-protein interaction networks and subnetworks in the biology of disease. Wiley Interdiscip Rev Syst Biol Med 3: 357-367, 2011.

16. Wu Y, Jing R, Jiang L, Jiang Y, Kuang Q, Ye L, Yang L, Li Y and $\mathrm{Li} \mathrm{M}$ : Combination use of protein-protein interaction network topological features improves the predictive scores of deleterious non-synonymous single-nucleotide polymorphisms. Amino Acids 46: 2025-2035, 2014.

17. Ma X, Gao L and Tan K: Modeling disease progression using dynamics of pathway connectivity. Bioinformatics 30: 2343-2350, 2014.

18. Benjamini Y and Hochberg Y: Controlling the false discovery rate: A practical and powerful approach to multiple testing. J R Stat Soc B 57: 289-300, 1995.

19. Sauer U, Lasko DR, Fiaux J, Hochuli M, Glaser R, Szyperski T, Wüthrich K and Bailey JE: Metabolic flux ratio analysis of genetic and environmental modulations of Escherichia coli central carbon metabolism. J Bacteriol 181: 6679-6688, 1999.

20. Harman D: The free radical theory of aging: Effect of age on serum copper levels. J Gerontol 20: 151-153, 1965.

21. Park CB and Larsson NG: Mitochondrial DNA mutations in disease and aging. J Cell Biol 193: 809-818, 2011.

22. Safdar A, Bourgeois JM, Ogborn DI, Little JP, Hettinga BP, Akhtar M, Thompson JE, Melov S, Mocellin NJ, Kujoth GC, et al: Endurance exercise rescues progeroid aging and induces systemic mitochondrial rejuvenation in mtDNA mutator mice. Proc Natl Acad Sci USA 108: 4135-4140, 2011.

23. Baker DJ, Dawlaty MM, Wijshake T, Jeganathan KB, Malureanu L, van Ree JH, Crespo-Diaz R, Reyes S, Seaburg L, Shapiro V, et al: Increased expression of BubR1 protects against aneuploidy and cancer and extends healthy lifespan. Nat Cell Biol 15: 96-102, 2013.

24. Blasco MA: Telomere length, stem cells and aging. Nat Chem Biol 3: 640-649, 2007.

25. Jaskelioff M, Muller FL, Paik JH, Thomas E, Jiang S, Adams AC, Sahin E, Kost-Alimova M, Protopopov A, Cadiñanos J, et al: Telomerase reactivation reverses tissue degeneration in aged telomerase-deficient mice. Nature 469: 102-106, 2011.

26. Finkel T and Holbrook NJ: Oxidants, oxidative stress and the biology of ageing. Nature 408: 239-247, 2000.

27. Mattson MP: Calcium and neurodegeneration. Aging Cell 6: 337-350, 2007.

28. Ristow M and Schmeisser S: Extending life span by increasing oxidative stress. Free Radic Biol Med 51: 327-336, 2011.

29. Zolezzi JM, Silva-Alvarez C, Ordenes D, Godoy JA, Carvajal FJ, Santos MJ and Inestrosa NC: Peroxisome proliferator-activated receptor (PPAR) $\gamma$ and PPAR $\alpha$ agonists modulate mitochondrial fusion-fission dynamics: Relevance to reactive oxygen species (ROS)-related neurodegenerative disorders? PLoS One 8: e64019, 2013.

30. Senovilla L, Vitale I, Martins I, Tailler M, Pailleret C, Michaud M, Galluzzi L, Adjemian S, Kepp O, Niso-Santano M, et al: An immunosurveillance mechanism controls cancer cell ploidy. Science 337: 1678-1684, 2012.

31. Barzilai N, Huffman DM, Muzumdar RH and Bartke A: The critical role of metabolic pathways in aging. Diabetes 61: 1315-1322, 2012.

32. Adler AS, Sinha S, Kawahara TLA, Zhang JY, Segal E and Chang HY: Motif module map reveals enforcement of aging by continual NF-kappaB activity. Genes Dev 21: 3244-3257, 2007.

33. Tilstra JS, Robinson AR, Wang J, Gregg SQ, Clauson CL, Reay DP, Nasto LA, St Croix CM, Usas A, Vo N, et al: NF-кB inhibition delays DNA damage-induced senescence and aging in mice. J Clin Invest 122: 2601-2612, 2012.

This work is licensed under a Creative Commons Attribution-NonCommercial-NoDerivatives 4.0 International (CC BY-NC-ND 4.0) License. 